\title{
Evaluating the relationship between quality measure adherence definitions and economic outcomes in commercial health plans: a retrospective diabetes cohort study
}

\author{
Chanadda Chinthammit, BSPharm, MS, PhD; David R Axon, PhD, MPharm, MS; Lea Mollon, PharmD; \\ Ann M Taylor, MPH, MCHES; Matthew Pickering, PharmD, RPh; Heather Black, PhD; \\ Terri Warholak, PhD, RPh, CPHQ, FAPhA; and Patrick J Campbell, PhD, PharmD, RPh
}

\section{What is already known about this subject}

- Diabetes is a prevalent chronic condition in the United States that results in considerable morbidity and mortality, frequent use of the health care system, and high health care expenditures.

- Adherence to noninsulin antidiabetic medication can help reduce health care system use and expenditures, while also improving health outcomes.

- Medication use quality measures, such as the Pharmacy Quality Alliance Proportion of Days Covered: Diabetes All Class adherence measure, have been developed and endorsed to improve adherence to noninsulin antidiabetic medications; however, their use in a commercial population over a 1-year time frame has not been assessed.

\section{What this study adds \\ - In multivariable analyses, adherence to noninsulin antidiabetic medications was associated with fewer inpatient visits and more outpatient visits and lower inpatient and total health care expenditures within a 1-year time frame. \\ - Compared with nonadherent subjects in adjusted multivariable analyses, those who were adherent were associated with lower incremental per member per month expenditures for inpatient and total health care expenditures.}

\section{ABSTRACT}

BACKGROUND: Diabetes is a prevalent chronic condition in the United States that results in considerable morbidity and mortality, frequent use of the health care system, and high health care expenditures. Adherence to antidiabetic medications can help improve health outcomes and lower health care utilization and expenditures. The Pharmacy Quality Alliance (PQA) Proportion of Days Covered (PDC): Diabetes All Class medication adherence measure was developed and endorsed to improve adherence to noninsulin antidiabetic medications; however, it has not been assessed in a commercial

\begin{abstract}
Author affiliations
Chanadda Chinthammit, BSPharm, MS, PhD, University of Arizona College of Pharmacy, Tucson, and Eli Lilly and Company, Indianapolis, IN. David R Axon, PhD, MPharm, MS; Lea Mollon, PharmD; Ann M Taylor, $\mathrm{MPH}, \mathrm{MCHES}$; and Terri Warholak, PhD, $\mathrm{RPh}, \mathrm{CPHQ}$, FAPhA, University of Arizona College of Pharmacy, Tucson. Matthew Pickering, PharmD, RPh, Pharmacy Quality Alliance, Alexandria, VA, and Heather Black, PhD, Merck \& Co., Kenilworth, NJ. Patrick J Campbell, PhD, PharmD, RPh, University of Arizona College of Pharmacy, Tucson, and Pharmacy Quality Alliance, Alexandria, VA.
\end{abstract}

AUTHOR CORRESPONDENCE:

David R Axon, 520.621.5961;

axon@pharmacy.arizona.edu

J Manag Care Spec Pharm. 2021;27(1):64-72

Copyright $@ 2021$, Academy of Managed Care Pharmacy. All rights reserved.

population of diabetes patients over a 1-year time frame.

OBJECTIVE: To determine the association between adherence, as defined in the PQA medication adherence measures, and health care utilization and expenditure among commercially insured individuals using antidiabetic medications. 
METHODS: This 1-year retrospective study evaluated a cohort of individuals from IBM MarketScan Research Databases (2009-2015) with noninsulin antidiabetic medications. Eligible study subjects included adults (aged $\geq 18$ years at index date) with continuous enrollment in their health plans for 6 months before (i.e., baseline period) and 12 months after (i.e., study period) the index date and $\geq 2$ prescriptions dispensed for any medication included in the PQA PDC Diabetes All Class medication adherence measure, with at least 150 days between the first and last fill during the study period. The index date was defined as the first fill for a medication included in the PQA PDC Diabetes All Class adherence measure after a 180-day baseline period. Generalized linear models with log link and gamma distribution (expenditure) or negative binomial distribution (utilization) assessed relationships between adherence ( $\geq 80 \%$ PDC) and health care utilization and expenditure while adjusting for potential confounders. Cost ratios (CR) and rate ratios (RR) were computed using beta coefficients. Cohort characteristics were compared using t-tests, Wilcoxon rank sum tests, or chi-square tests with an alpha level of 0.001 set a priori.

RESULTS: A total of $1,576,112$ individuals were eligible; of these, $1,028,176$ (65.2\%) were adherent. Significant differences in demographic characteristics were observed between adherent and nonadherent groups $(P<0.001)$. Multivariable analyses demonstrated that adherence was associated with the following: (a) 16.6\% fewer inpatient $(\mathrm{RR}=0.834,95 \% \mathrm{Cl}=0.819-0.850)$ and $3.6 \%$ more outpatient service visits $(R R=1.036,95 \% \mathrm{Cl}=1.032-1.039)$ and (b) $16.8 \%$ lower inpatient expenditures ( $\mathrm{CR}=0.833,95 \% \mathrm{Cl}=0.829-0.836) ; 2.6 \%$ lower outpatient expenditures ( $\mathrm{CR}=0.974,95 \% \mathrm{Cl}=0.970-0.978) ; 16.4 \%$ higher prescription drug expenditures ( $\mathrm{CR}=1.164,95 \% \mathrm{Cl}=1.159-1.169)$; and $4.2 \%$ lower total $(\mathrm{CR}=0.958,95 \% \mathrm{Cl}=0.954-0.962)$ expenditures. Adherent subjects were associated with lower incremental per member per month expenditures for inpatient (-\$31.74), outpatient (-\$10.09), and total (-\$30.82) expenditures, yet higher prescription drug expenditures (\$25.60) compared with nonadherent subjects.

CONCLUSIONS: Adherence to noninsulin antidiabetic medications was associated with more outpatient and fewer inpatient visits, as well as lower total expenditures compared with nonadherence.

Diabetes is a serious chronic condition affecting an estimated 34 million people in the United States alone. ${ }^{1}$ Most diabetes cases (90\%-95\%) are classified as having type 2 diabetes. Prevalence increases with age, and over one quarter of individuals aged 65 years and older have the disease. ${ }^{1}$ Furthermore, diabetes is costly-medical expenditures for those with the disease are 2.3 times higher than for those without. ${ }^{2}$ Overall, diabetes accounted for an estimated $\$ 327$ billion in total health care costs (direct and indirect), with \$237 billion in direct costs largely due to hospital inpatient care, prescription medications, diabetic supplies, provider visits, and long-term care in 2017. ${ }^{2}$ Despite the associated morbidity, mortality, and costs, adherence to oral antidiabetic medications is associated with better outcomes and lower health care utilization.

Medication adherence corresponds to decreases in glycated hemoglobin A1c (HbA1c), a marker of glycemic control that is associated with improved long-term outcomes..$^{3-5}$ Further, adherence to oral antidiabetic medications is associated with lower health care costs. ${ }^{6}$ Specifically, medication adherence among patients with diabetes can result in cost savings, whereas nonadherence to oral antidiabetic medications is associated with increased health care utilization and consequential health care costs..$^{7-9}$ Furthermore, new advances in diabetes management often require the use of several medications and structured treatment plans, including strategies to improve medication adherence that may appreciably improve short- and long-term health. ${ }^{10-13}$

In an effort to measure adherence, payers may use tested and endorsed medication use quality measures developed by the Pharmacy Quality Alliance (PQA), a multistakeholder, nonprofit national quality organization that develops medication use quality measures. These measures are also key performance drivers in the Centers for Medicare \& Medicaid Services' (CMS) Star Rating System for Medicare Advantage Prescription Drug plans and standalone Prescription Drug Plans. The PQA Proportion of Days Covered (PDC) Diabetes All Class medication adherence measure includes multiple diabetes drug classes (excluding insulin) and assesses the percentage of patients who are covered by at least 1 of the antidiabetic medication classes within a measurement year. ${ }^{14} \mathrm{PQA}$ uses the PDC methodology to measure adherence. PDC represents the proportion of days that the medication is available to an individual during the measurement period over the total number of days in the measurement period.

Finally, the link between poor medication adherence and increased use, negative clinical outcomes, costs, and mortality is well established..$^{-9,911,15}$ To date, the association between adherence, as defined in PQA adherence measures, and health care utilization and expenditure in a commercial population over a 1-year period has not been assessed. To address this gap, this study's aim was to describe the short-term (i.e., 1 year) relationship between noninsulin antidiabetic medication adherence and health care utilization and expenditure.

\section{Methods}

\section{DATA SOURCE AND STUDY DESIGN}

This retrospective cohort study used a subset of IBM MarketScan Commercial Claims and Encounters Research 
Databases (January 1, 2009-December 31, 2015) to assess the relationship between noninsulin antidiabetic medication adherence and economic outcomes among a commercially insured patient population. Deidentified data elements included subject demographics, enrollment details, medical diagnoses, and procedures, as well as prescription, inpatient, and outpatient administrative claims. The University of Arizona Institutional Review Board approved this retrospective study.

\section{ELIGIBILITY CRITERIA}

Subject eligibility was informed by the 2015 PQA PDC Diabetes All Class medication adherence measure specifications. ${ }^{14}$ The 2015 measure specifications includes adults aged 18 years and older on the first day of the measurement year who have continuous pharmacy benefit enrollment and 2 or more prescription claims for noninsulin, oral, or injectable antidiabetic medications with at least 150 days between the first and last fill. The measure excludes individuals with 1 or more prescription claims for insulin and those with a diagnosis of end-stage renal disease during the measurement year.

For the purposes of this study, the index date was defined as the first fill for a medication included in the PQA PDC Diabetes All Class adherence measure after a 180-day baseline period. Adults (aged 18 years or older at the index date) were included in the cohort if they had the following: (a) continuous enrollment in medical and pharmacy benefits for 6 months before (i.e., baseline period) and 12 months after (i.e., study period) the index date and (b) at least 2 prescriptions dispensed for any medication included in the PQA PDC Diabetes All Class medication adherence measure, with at least 150 days between the first and last fill during the study period.

Individuals were excluded if they had 1 or more prescription claims for an insulin product (identified using PQA National Drug Code (NDC) value sets and pharmacy claims data) or had a diagnosis of end-stage renal disease, based on the International Classification of Diseases, Ninth Revision, Clinical Modification (ICD-9-CM) code of 585.6 during the 12-month study period.

\section{OUTCOME VARIABLES}

This study investigated the association of adherence, as defined in the PQA PDC Diabetes All Class adherence measure,${ }^{14}$ with economic outcomes from the payer perspective (i.e., health care resource utilization and costs). Health care utilization consisted of the number of all-cause inpatient and outpatient visits during the 1-year study period. All-cause, nonreversed, paid health care payer claim costs, including inpatient, outpatient, prescription drug, and total health care expenditure for the study period, were calculated; all costs were adjusted to 2015 U.S. dollars using the U.S. Department of Labor Consumer Price Index. ${ }^{16}$

\section{INDEPENDENT VARIABLE}

The key independent variable was adherence status, measured via the PQA PDC methodology for noninsulin antidiabetic medications (i.e., biguanides, sulfonylureas, thiazolidinediones, dipeptidyl peptidase-4 inhibitors, incretin mimetics, meglitinides, and sodium glucose cotransporter-2 inhibitors) ${ }^{17,18}$ PQA PDC Diabetes All Class specifications and NDC value sets from 2015 were used to identify eligible oral and noninsulin injectable medications to include in the PDC calculation. PDC was calculated by counting the number of days that the individual was covered by at least 1 drug in the measure based on the prescription fill date and days supply. Patients were classified as adherent if their PDC was $\geq 80 \%$.

\section{COVARIATES}

Potential confounders or risk factors for health care utilization and expenditure were included as covariates in the statistical models. With the exception of monthly average number of noninsulin antidiabetic medications, all of these covariates were measured during the baseline period: age (in years at index date), Deyo-Charlson Comorbidity Index (CCI), gender, geographic region, insurance plan type, monthly average number of chronic medications, and current or new user of antidiabetic medications. Patients were categorized as "current users" if they filled a prescription for an included antidiabetic medication during the baseline period, whereas "new users" first filled an included antidiabetic medication on the index date (i.e., they had no noninsulin antidiabetic medication during the baseline period). The monthly average number of chronic medications (prescription days supply greater than or equal to 28) was calculated to represent medication burden. The monthly average number of unique antidiabetic medication products used during the study period was calculated to differentiate between treatment intensity (i.e., more medications indicated greater treatment intensity).

\section{STATISTICAL ANALYSIS}

Generalized linear models (GLM) were used to test the relationships between noninsulin antidiabetic medication adherence and the outcomes of interest, with adjustment for the covariates. A GLM with a log link and negative binomial distribution was used to assess the relationship between adherence and health care utilization. A GLM with log link and gamma distribution assessed the relationship between adherence and health care expenditures. Beta coefficients, 


\section{FIGURE 1 Cohort Flowchart Diagram (2009-2015)}

Individuals with prescription drug claims data $\mathrm{N}=16,225,093$

Excluded those:

- with no noninsulin antidiabetic medication observations, $n=12,173,755^{\text {a }}$

- aged less than 18 years at the index date, $n=452,662$

- with less than 2 noninsulin antidiabetic medication fills during the measurement period, $n=497,989$

- with less than 150 days between and last fill during the measurement period, $n=766,545$

- without continuous enrollment, $n=493,177$

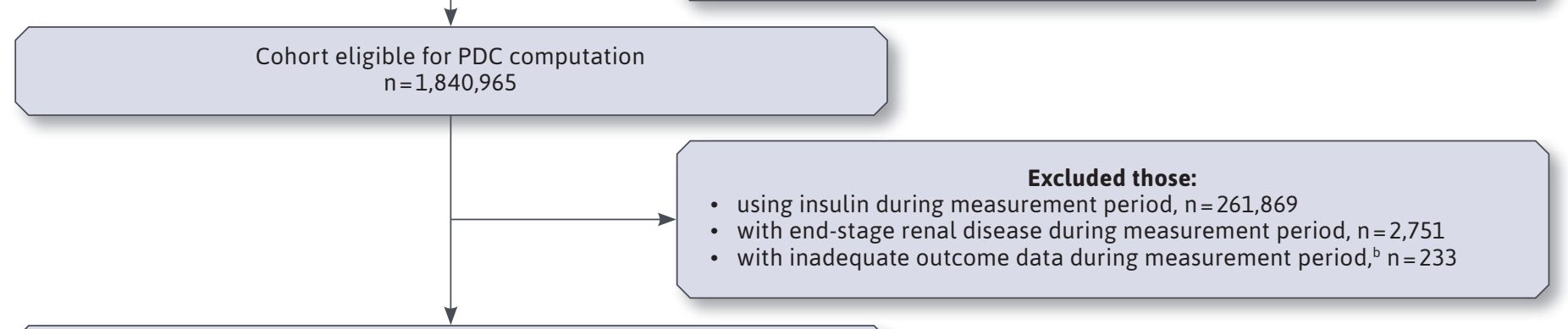

Final cohort for 1-year measurement period

$\mathrm{n}=1,576,112$

aNoninsulin oral and injectable antidiabetic medications included in the PQA PDC: Diabetes All Class adherence measure.

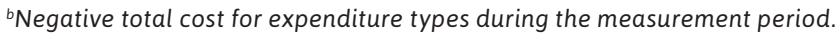

$P D C=$ proportion of days covered; $P Q A=$ Pharmacy Quality Alliance.

generated from GLMs, were used to compute the cost ratios (CRs) and risk ratios (RRs) to demonstrate differences in health care utilization and expenditures between the adherent and nonadherent groups, using semi-logarithmic equations. ${ }^{19}$

Average expenditure per member per month (PMPM) was calculated to demonstrate differences in each type of expenditure (i.e., inpatient, outpatient, prescription drug, and total) between the adherent and nonadherent groups. Subject characteristics were assessed using t-tests or Wilcoxon rank sum tests for continuous variables, and chi-square tests were used for categorical variables. An alpha level of 0.001 was set a priori for all analyses. All analyses were conducted using SAS version 9.4 (SAS Institute, Cary, NC).

\section{Results}

Of the 16.2 million individuals with prescription claims data in the subset of IBM MarketScan Commercial Claims and
Encounters Research Databases (January 1, 2009-December 31, 2015), 1,840,965 adults were eligible for PDC calculation. After applying the exclusion criteria, 1,576,112 individuals were included in the study. Figure 1 outlines the patient selection process details.

\section{DESCRIPTIVE ANALYSES}

A total of 1,028,176 (65.2\%) subjects were classified as adherent. The adherent group had a mean age (SD) of 53.2 years (8.4), and roughly half (53.8\%) were males from the South (42.3\%) and North Central (21.9\%) regions of the United States, with the vast majority insured by preferred provider organizations (62.1\%) and health maintenance organizations (14.8\%). Low CCI scores were prevalent, with only $1.3 \%$ of the adherent group having a score of 5 or greater. The adherent group took a median of 3.3 (interquartile range $=$ 3.5) chronic medications per month. A majority of individuals in the adherent group were identified as current oral 


\section{TABLE 1 Characteristics of Study Subjects}

\begin{tabular}{|c|c|c|c|c|}
\hline \multirow{3}{*}{$\begin{array}{l}\text { Characteristic } \\
\text { Age (years), mean (SD) }\end{array}$} & \multicolumn{4}{|c|}{ Total $(\mathrm{N}=1,576,112)$} \\
\hline & \multicolumn{2}{|c|}{$\begin{array}{c}\text { Adherent } \\
\mathrm{n}=1,028,176 \\
(65.2 \%)\end{array}$} & \multicolumn{2}{|c|}{$\begin{array}{c}\text { Nonadherent } \\
\mathbf{n}=547,936 \\
(34.8 \%)\end{array}$} \\
\hline & 53.2 & $(8.4)$ & 48.9 & $(10.4)$ \\
\hline Male gender, n (\%) & 553,580 & $(53.8)$ & 239,769 & $(43.8)$ \\
\hline \multicolumn{5}{|l|}{ Region, n (\%) } \\
\hline Northeast & 176,149 & $(17.1)$ & 74,771 & $(13.6)$ \\
\hline North Central & 225,542 & $(21.9)$ & 107,946 & $(19.7)$ \\
\hline South & 435,417 & $(42.3)$ & 268,729 & $(49.0)$ \\
\hline West & 171,468 & $(16.7)$ & 85,420 & $(15.6)$ \\
\hline Unknown & 19,600 & $(1.9)$ & 11,070 & $(2.0)$ \\
\hline \multicolumn{5}{|l|}{ Plan type, n (\%) } \\
\hline Comprehensive & 23,952 & $(2.3)$ & 9,005 & $(1.6)$ \\
\hline Exclusive provider organization & 13,289 & (1.3) & 7,251 & (1.3) \\
\hline Health maintenance organization & 151,864 & $(14.8)$ & 82,036 & $(15.0)$ \\
\hline Point of service & 76,198 & (7.4) & 41,172 & $(7.5)$ \\
\hline Preferred provider organization & 638,916 & $(62.1)$ & 341,103 & $(62.3)$ \\
\hline Point of service with capitation & 4,969 & $(0.5)$ & 2,666 & $(0.5)$ \\
\hline Consumer-directed health plan & 39,737 & (3.9) & 24,599 & $(4.5)$ \\
\hline High deductible health plan & 19,520 & $(1.9)$ & 11,910 & $(2.2)$ \\
\hline Unknown & 59,731 & $(5.8)$ & 28,194 & $(5.2)$ \\
\hline \multicolumn{5}{|l|}{ Charlson Comorbidity Index, n (\%) } \\
\hline 0 & 246,134 & $(23.9)$ & 195,992 & $(35.8)$ \\
\hline 1 & 604,167 & $(58.8)$ & 278,739 & $(50.9)$ \\
\hline 2 & 83,160 & $(8.1)$ & 39,462 & $(7.2)$ \\
\hline 3 & 68,571 & $(6.7)$ & 23,954 & $(4.4)$ \\
\hline 4 & 12,754 & $(1.2)$ & 4,809 & $(0.9)$ \\
\hline $5+$ & 13,390 & (1.3) & 4,980 & $(0.9)$ \\
\hline New user, n (\%) & 405,939 & $(39.5)$ & 341,871 & $(62.4)$ \\
\hline $\begin{array}{l}\text { Average number of chronic medications in baseline, } \\
\text { median (IQR) }\end{array}$ & 3.3 & $(3.5)$ & 1.7 & $(2.5)$ \\
\hline $\begin{array}{l}\text { Average number of antidiabetic medications in study } \\
\text { period, median (IQR) }\end{array}$ & 1.2 & $(0.7)$ & 0.6 & $(0.3)$ \\
\hline Outpatient utilization, ${ }^{a}$ mean (SD) & 14.3 & $(13.7)$ & 13.3 & $(13.4)$ \\
\hline Inpatient utilization, a mean (SD) & 0.1 & $(0.5)$ & 0.1 & $(0.6)$ \\
\hline Outpatient expenditure, ${ }^{\mathrm{b}}$ mean (SD) & 4,728 & $27,436)$ & 4,637 & $12,747)$ \\
\hline Inpatient expenditure, ${ }^{\mathrm{b}}$ mean (SD) & 2,047 & 13,176) & 2,273 & $15,609)$ \\
\hline Drug expenditure, ${ }^{\mathrm{b}}$ mean (SD) & 3,384 & $(6,618)$ & 1,874 & $(4,982)$ \\
\hline Total expenditure, ${ }^{\mathrm{b}}$ mean (SD) & 10,159 & $32,765)$ & 8,785 & $23,668)$ \\
\hline
\end{tabular}

Note: All subject characteristics were significant at the $P<0.001$ level unless otherwise noted. a Outpatient utilization includes laboratory tests, office visits, and other outpatient services; inpatient utilization includes inpatient admissions, emergency department use, and other inpatient services. ${ }^{b}$ Expenditure adjusted to 2015 U.S. dollars.

$I Q R=$ interquartile range. antidiabetic medication users, while $39.5 \%$ were considered new users.

A total of $547,936(34.8 \%)$ subjects were classified as nonadherent. The nonadherent group had a mean (SD) age of 48.9 years (10.4), and $43.8 \%$ were males from the South $(49.0 \%)$ and North Central (19.7\%) regions of the United States, with the vast majority insured by preferred provider organizations (62.3\%) and health maintenance organizations (15.0\%). Low CCI scores were prevalent, with $0.9 \%$ of the nonadherent group having a score of 5 or greater. The nonadherent group took a median of 1.7 (interquartile range $=2.5$ ) chronic medications per month. A majority of individuals in the nonadherent group were identified as new users of oral antidiabetic medication (62.4\%). Table 1 presents characteristics reported for adherent and nonadherent subjects.

During the 1-year study period, before adjusting for potential confounding variables, the average (SD) number of inpatient and outpatient visits was $0.1(0.5)$ and 14.3 (13.7) and 0.1 (0.6) and 13.3 (13.4) in the adherent group and nonadherent groups, respectively. For adherent individuals, before adjusting for potential confounding variables, the average inpatient expenditure was lower (\$2,047 vs. \$2,273), yet the average outpatient expenditure ( $\$ 4,728$ vs. $\$ 4,637)$, prescription drug expenditure (\$3,384 vs. $\$ 1,874)$, and total expenditure $(\$ 10,159$ vs. $\$ 8,785)$ was higher than for the nonadherent group.

\section{MULTIVARIABLE ANALYSES}

In adjusted multivariable analyses, adherence was associated with $16.6 \%$ fewer inpatient service visits $(\mathrm{RR}=0.834,95 \% \mathrm{CI}=0.819-0.850)$ and $3.6 \%$ more outpatient service visits $(\mathrm{RR}=1.036,95 \% \mathrm{CI}=1.032-1.039) \mathrm{com}^{-}$ pared with nonadherence. Adherent individuals were also associated with $16.8 \%$ lower inpatient $(\mathrm{CR}=0.832,95 \%$ 


\section{TABLE 2}

\section{Adjusted Results from Generalized Linear Models for Health Care Utilization and Expenditure of Study Subjects}

\begin{tabular}{l|cc|c}
\hline \hline Utilization $^{\mathrm{a}}$ & \multicolumn{2}{|c|}{ RR $(95 \% \mathrm{CI})$} & Percent change $^{\mathrm{c}}$ \\
\hline Inpatient & 0.834 & $(0.819-0.850)$ & -16.6 \\
\hline Outpatient & 1.036 & $(1.032-1.039)$ & 3.6 \\
\hline Expenditure $^{\mathrm{b}}$ & \multicolumn{2}{|c|}{ CR $(95 \% \mathrm{CI})$} & Percent change $^{\mathrm{c}}$ \\
\hline Inpatient & 0.832 & $(0.829-0.836)$ & -16.8 \\
\hline Outpatient & 0.974 & $(0.970-0.978)$ & -2.6 \\
\hline Prescription drug & 1.164 & $(1.159-1.169)$ & 16.4 \\
\hline Total & 0.958 & $(0.954-0.962)$ & -4.2 \\
\hline
\end{tabular}

a Health care utilization was assessed using a generalized linear model with log link and negative binomial distribution adjusted for age, sex, plan type, region, Deyo-Charlson Comorbidity Index, medication use status, average number of chronic medications used at baseline per month, and average number of antidiabetic mediations used during the study period per month. ${ }^{b}$ Health care expenditure was assessed using a generalized linear model with log link and gamma distribution adjusted for age, sex, plan type, region, Deyo-Charlson Comorbidity Index, medication use status, average number of chronic medications used at baseline per month, and average number of antidiabetic mediations used during the study period per month.

cPercent change indicated the percent difference in the adherent group compared with the nonadherent group.

$\mathrm{Cl}=$ confidence interval; $\mathrm{CR}=$ cost ratio; $\mathrm{R} R=$ risk ratio.

$\mathrm{CI}=0.829-0.836)$ and $2.6 \%$ lower outpatient $(\mathrm{CR}=0.974$, $95 \% \mathrm{CI}=0.970-0.978)$ health care expenditures, as well as $16.4 \%$ higher prescription drug expenditures $(\mathrm{CR}=1.164,95 \%$ $\mathrm{CI}=1.159-1.169)$ compared with nonadherent individuals. For total health care expenditure, adherence was associated with $4.2 \%$ lower expenditures compared wirh nonadherence $(\mathrm{CR}=0.958,95 \% \mathrm{CI}=0.954-0.962)$. Table 2 contains more information regarding the association between adherence and health care utilization and expenditure.

The average PMPM incremental cost of adherence is depicted in Figure 2. Based on multivariable model results, adherent patients were associated with savings of \$31.74 in inpatient expenditure, savings of \$10.09 in outpatient expenditure, yet had $\$ 25.60$ more in prescription drug expenditure compared with nonadherent patients. Adherent individuals were associated with total of $\$ 30.82$ PMPM health care savings compared with nonadherence.

\section{Discussion}

This study assessed the association of adherence to noninsulin antidiabetic medications, as defined in PQA medication adherence quality measures, with health care utilization and expenditures within a commercially insured U.S. adult population. Furthermore, it is one of the first to assess economic outcomes associated with adherence as it is calculated in the PQA PDC Diabetes All Class medication adherence measure. These results fill important gaps in the published literature, substantiating that (a) large effects are associated between adherence and health care service use and subsequent health care expenditure among a commercially covered diabetic population and (b) economic associations of adherence can be observed rapidly (i.e. within 1 year).

The relationship between oral antidiabetic medication adherence and economic outcomes (utilization and expenditures) has been studied; however, discrepancies exist in the current literature regarding definitions of adherence, approaches, and outcomes. Multiple studies have reported an association of lower utilization ${ }^{20-23}$ and expenditures ${ }^{13,22,24}$ among individuals who are adherent to their oral antidiabetic medications. To date, some have used medication possession ratio (MPR) as an adherence measure, reporting that values less than $80 \%$ were associated with worse clinical outcomes ${ }^{5}$ and increased hospital, emergency room, and physician visits. ${ }^{25}$ Moreover, others have examined the relationships between adherence to glucose-lowering agents, using PDC as a measure of adherence. ${ }^{26,27}$

Consistent with the current findings, other studies have reported that adherence to oral antidiabetic medications resulted in higher pharmacy costs yet lower medical costs than nonadherence. Recently, Curtis et al. (2017) used the IBM MarketScan Commercial Claims and Encounters database to examine patients with type 2 diabetes and the relationship between adherence to glucose-lowering agents and all-cause medical costs, acute care resource utilization, and acute complications. ${ }^{28}$ They found a significant reduction in acute care costs and resource utilization yet found no difference in all-cause medical costs over a 3-year period. ${ }^{28}$ In another study of the association between oral antidiabetic medication adherence and costs, adherence was also found to be associated with higher drug costs yet lower inpatient, outpatient, and total costs. ${ }^{29}$

While the current study findings are concordant with much of the previous literature on adherence, utilization, and expenditure, this study is the first to use PQA adherence measure specifications as a standard for defining adherence in a large sample of commercially insured beneficiaries over a 1-year period. Use of standard adherence definitions, value sets, and methodology can facilitate a more direct comparison of findings in the literature because of the myriad nuanced PDC and MPR approaches. ${ }^{30,31}$ Additionally, variation in selected medications and medication classes 


\section{FIGURE 2 Incremental Cost of Being Adherent Compared with Nonadherent PMPM}

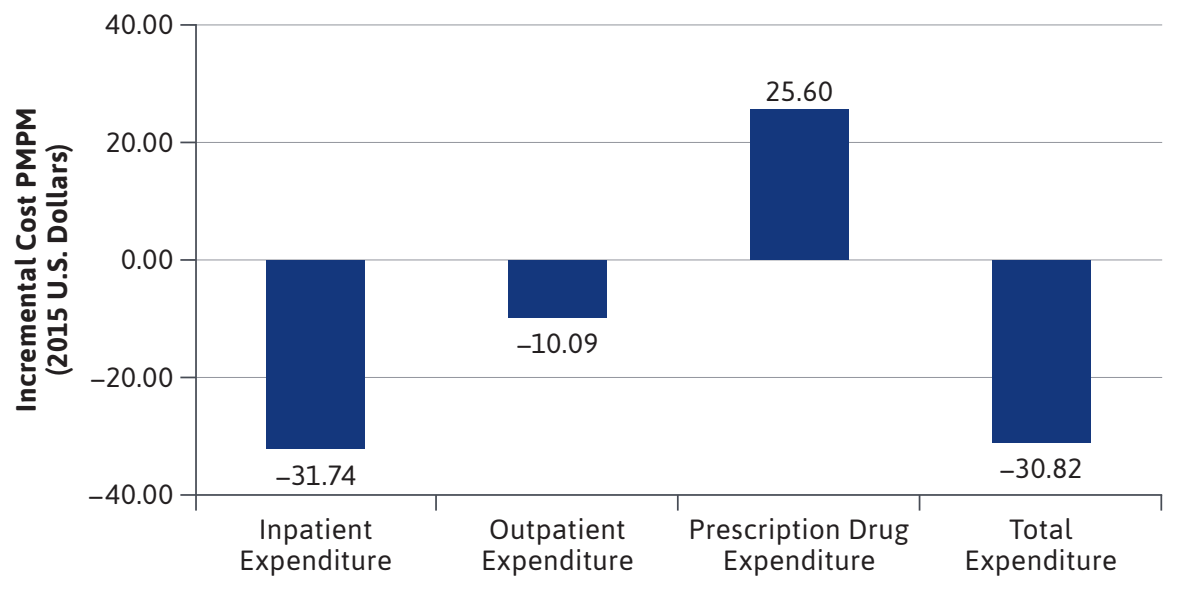

PMPM = per member per month.

included in adherence studies makes it more difficult to compare findings.

The PQA PDC Diabetes All Class adherence measure specifications detail the methodology of calculating PDC (i.e., by counting the days an individual was covered by at least 1 drug included in the measure based on the prescription fill date and days supply) and describe how and when to adjust for overlap of target medications (i.e., if prescriptions for the same target drug ingredient overlap, adjust the prescription start date to be the day after the previous fill has ended). This approach provides a more conservative estimate of adherence in instances of frequent medication switches and concomitant therapy with multiple drugs within a class..$^{30}$

The current findings add to the published literature by linking this quality measure specified antidiabetic medication adherence definition to a significant reduction in acute care expenditures, as well as a significant association with lower payer total health care expenditures. Therefore, commercial health insurance plans may consider adopting PQA measures to help improve quality, reduce costs, and improve outcomes among the commercially insured population. However, commercial insurance plans should also consider the cost of outof-pocket payments made by patients, since a previous study showed that out-of-pocket pharmacy costs and adherence to noninsulin antidiabetic medications were negatively associated. ${ }^{32}$

This study corroborates findings from the previous literature regarding the association between antidiabetic medication adherence with lower total health care expenditures and utilization. ${ }^{31,33}$ In this study, the adherent group had lower associated health care utilization and costs than the nonadherent group. Furthermore, this study adds to the knowledge base that includes a large sample size using commercial claims data and employing the PQA adherence measure specifications (e.g., use of the PQA PDC Diabetes All Class measure PDC calculation, with the exclusion of patients taking insulin and patients with end-stage renal disease).

Finally, one of the most noteworthy findings is the potential for observing adherence effects on health care utilization and expenditures within a single year, compared with other studies that have reported similar effects over several years.

\section{LIMITATIONS}

This study had several limitations. The retrospective database design used administrative health insurance claims data intended for insurance reimbursement purposes; therefore, the potential for billing and coding errors exists. Furthermore, in the absence of confirmatory medical chart review, disease states and drug exposure misclassification and missing or inaccurate coding of administrative claims may have occurred. Medication exposure, based on filled prescriptions, is an indirect measure of actual behavior and may not accurately reflect actual medication adherence.

This database analysis may also have been subject to selection bias, whereby individuals in the adherent group were different from those in the nonadherent group. Multivariable analyses accounted for differences between groups; however, the demographic and clinical data were limited to those individuals who had administrative claims. To account for the risk of immortal time bias, individuals were categorized as new or current users, based on initial prescription fill during the study period.

The IBM Marketscan Research Database is a convenience sample of covered individuals. Additionally, patients with certain higher-cost conditions (e.g., liver disease, cancer, 
receiving palliative care) were included in the analysis, while others were excluded for certain medications and disease states (e.g., insulin, end-stage renal disease). Thus, the generalizability of these study results to other patient populations (e.g., those with other insurance types or no insurance, patients with diabetes taking insulin) are limited.

\section{Conclusions}

This large, retrospective database study is one of the first to assess medication adherence using the methodology used in the PQA PDC Diabetes All Class adherence measure. These results add a new finding to the published literature by showing that medication adherence was associated with lower health care resource utilization and cost within a single year's time frame. Yet, further investigation is warranted to study the associations of medication adherence among patients using insulin products.

\section{DISCLOSURES}

Funding was provided by grants from Pharmacy Quality Alliance, Merck \& Co. (Kenilworth, NJ), and SinfoniaRx. In addition, Chinthammit reports personal fees from Eli Lilly and Company, outside the submitted work. Axon reports grants from the American Association of Colleges of Pharmacy and the Arizona Department of Health Services, outside the submitted work. Taylor reports grants from the Arizona Department of Health Services, outside the submitted work. Warholak reports grants from Novartis and the Arizona Department of Health Services, outside the submitted work. Chinthammit and Campbell disclose that this work was completed during their employment at the University of Arizona.

This research was presented as a poster at the AMCP Annual Meeting 2019; March 25-28, 2019; San Diego, CA.

\section{REFERENCES}

1. Centers for Disease Control and Prevention. National diabetes statistics report, 2020. Accessed December 5, 2020. https://www.cdc.gov/diabetes/pdfs/ data/statistics/national-diabetes-statistics-report.pdf

2. American Diabetes Association. Economic costs of diabetes in the U.S. in 2017. Diabetes Care. 2018;41(5):917-28.

3. Schectman JM, Nadkarni MM, Voss JD. The association between diabetes metabolic control and drug adherence in an indigent population. Diabetes Care. 2002;25(6):1015-21.

4. Krapek K, King K, Warren SS, et al. Medication adherence and associated hemoglobin A1c in type 2 diabetes. Ann Pharmacother. 2004;38(9):1357-62.

5. Egede LE, Gebregziabher M, Echols C, Lynch CP. Longitudinal effects of medication nonadherence on glycemic control. Ann Pharmacother. 2014;48(5):562-70.

6. Krass I, Schieback P, Dhippayom T. Adherence to diabetes medication: a systematic review. Diabet Med. 2015;32(6).725-37.

7. Balkrishnan R, Rajagopalan R, Camacho FT, Huston SA, Murray FT, Anderson RT. Predictors of medication adherence and associated health care costs in an older population with type 2 diabetes mellitus: a longitudinal cohort study. Clin Ther. 2003;25(11):2958-71.

8. Shenolikar RA, Balkrishnan R, Camacho FT, Whitmire JT, Anderson RT. Comparison of medication adherence and associated health care costs after introduction of pioglitazone treatment in African Americans versus all other races in patients with type 2 diabetes mellitus: a retrospective data analysis. Clin Ther. 2006;28(8):1199-207.

9. Egede LE, Gebregziabher M, Dismuke CE, et al. Medication nonadherence in diabetes: longitudinal effects on costs and potential cost savings from improvement. Diabetes Care. 2012;35(12):2533-39.
10. Hashmi NR, Khan SA. Adherence to diabetes mellitus treatment guidelines from theory to practice: the missing link. J Ayub Med Coll Abbottabad. 2016;28(4):802-08.

11. DiMatteo MR, Giordani PJ, Lepper HS, Croghan TW. Patient adherence and medical treatment outcomes: a meta-analysis. Med Care. 2002;40(9):794-811.

12. Sapkota S, Brien JA, Greenfield JR, Aslani P. A systematic review of interventions addressing adherence to anti-diabetic medications in patients with type 2 diabetes-components of interventions. PLoS One. 2015;10(6):e0128581.

13. Sokol MC, McGuigan KA, Verbrugge RR, Epstein RS. Impact of medication adherence on hospitalization risk and healthcare cost. Med Care. 2005;43(6):521-30.

14. Pharmacy Quality Alliance. PQA quality measures. Accessed December 5 , 2020. https://www.pqaalliance.org/ pqa-measures

15. Lipton HL, Bird JA. The impact of clinical pharmacists' consultations on geriatric patients' compliance and medical care use: a randomized controlled trial. Gerontologist. 1994;34(3):307-15.

16. U.S. Bureau of Labor Statistics. Consumer Price Index (CPI) databases. Acccessed December 5, 2020. https:// www.bls.gov/cpi/data.htm

17. Nau DP. Proportion of days covered (PDC) as a preferred method of measuring medication adherence. Accessed December 5, 2020. https://sep.yimg.com/ ty/cdn/epill/pdcmpr.pdf

18. Centers for Medicare \& Medicaid Services. Part C and D performance data. Accessed December 5, 2020. https://www.cms.gov/medicare/ prescription-drug-coverage/prescriptiondrugcovgenin/performancedata.html

19. Halvorsen R, Palmquist R. The interpretation of dummy variables in semilogarithmic equations. Am Econ Rev. 1980;70(3):474-75. 
20. Jha AK, Aubert RE, Yao J, Teagarden JR, Epstein RS. Greater adherence to diabetes drugs is linked to less hospital use and could save nearly $\$ 5$ billion annually. Health Aff (Millwood). 2012;31(8):1836-46.

21. Juarez DT, Tan C, Davis J, Mau M. Factors affecting sustained medication adherence and its impact on health care utilization in patients with diabetes. J Pharm Health Serv Res. 2013;4(2):89-94.

22. Roebuck MC, Liberman JN, Gemmill-Toyama M, Brennan TA. Medication adherence leads to lower health care use and costs despite increased drug spending. Health Aff (Millwood). 2011;30(1):91-99.

23. Wong ES, Bryson CL, Hebert PL, Liu CF. Estimating the impact of oral diabetes medication adherence on medical costs in VA. Ann Pharmacother. 2014;48(8):978-85.

24. Hansen RA, Farley JF, Droege M, Maciejewski ML. A retrospective cohort study of economic outcomes and adherence to monotherapy with metformin, pioglitazone, or a sulfonylurea among patients with type 2 diabetes mellitus in the United States from 2003 to 2005 . Clin Ther. 2010;32(7):1308-19.
25. DiBonaventura M, Wintfeld N, Huang J, Goren A. The association between nonadherence and glycated hemoglobin among type 2 diabetes patients using basal insulin analogs. Patient Prefer Adherence. 2014;8:873-82.

26. Hagen SE, Wright DW, Finch R, Talamonti WJ, Edington DW. Impact of compliance to oral hypoglycemic agents on short-term disability costs in an employer population. Popul Health Manag. 2014;17(1):35-41.

27. Stuart BC, Dai M, Xu J, Loh FH, Dougherty J. Does good medication adherence really save payers money? Med Care. 2015;53(6):517-23.

28. Curtis SE, Boye KS, Lage MJ, Garcia-Perez LE. Medication adherence and improved outcomes among patients with type 2 diabetes. Am J Manag Care. 2017;23(7):e208-e214.

29. Men J, Casciano R, Lee Y-C, et al. Effect of diabetes treatment-related attributes on costs to type 2 diabetes patients in real-word population. J Manag Care Spec Pharm. 2017;23(4):446-52. doi: 10.18553/jmcp.2017.23.4.446
30. Raebel MA, Schmittdiel J, Karter AJ, et al. Standardizing terminology and definitions of medication adherence and persistence in research employing electronic databases. Med Care. 2013;51 (8 Suppl 3):S11-21.

31. Kennedy-Martin T, Boye KS, Peng X. Cost of medication adherence and persistence in type 2 diabetes mellitus: a literature review. Patient Prefer Adherence. 2017;11:1103-17.

32. Bibeau WS, Fu H, Taylor AD, Kwan AYM. Impact of out-of-pocket pharmacy costs on branded medication adherence among patients with type 2 diabetes. J Manag Care Spec Pharm. 2016;22(11):1338-47. doi: 10.18553/jmcp.2016.22.11.1338

33. Shenolikar RA, Balkrishnan R. Oral antidiabetes medication adherence and health care utilization among Medicareenrolled type 2 diabetic patients beginning monotherapy. Diabetes Care. 2008;31(2):e5. 